\title{
Implementation of Wimax Simulator in Simulink
}

\author{
${ }^{1}$ Mirnall Bansal , ${ }^{2}$ Maninder Kaur,${ }^{3}$ Mohinder Pal Joshi \\ ${ }^{1}$ E.C.E. Department, D.I.E.T. Kharar, Mohali, PTU Jalandhar, Punjab, India \\ ${ }^{2}$ E.C.E. Department, D.I.E.T. Kharar, Mohali, PTU Jalandhar, Punjab, India \\ ${ }^{3}$ E.C.E. Department, S.L.I.E.T, Longowal, Punjab, India
}

\begin{abstract}
The needs of high speed broadband wireless access at lower cost and easy deployment to meet the modern mobile services leads in the emergence of an another IEEE standard called Worldwide Interoperability for Microwave Access (WiMAX). The limitations of conventional Broadband wireless access have been overcome with the scalable features of WiMAX. The main purpose of this paper is to evaluate, analyze and compare the performance of a WiMAX under different data rate. For this purpose a simulation model of WiMAX transmitter and Receiver has been designed using Simulink in MATLAB. The model presented in this paper using, outer coding and interleaving, inner coding and interleaving, constellation and mapping blocks of encoder and decoder are implemented. The performance of transmitted data depends on parameters like Bit error rate (BER).
\end{abstract}

Keywords: - Wimax IEEE802.16 encoder, Convolution Interleaving, Modulation, Ofdm, and BER.

\section{INTRODUCTION}

WiMAX, the IEEE 802.16e standard has brought a revolution in wireless broadband technology. Fixed Broadband Wireless Access (FBWA) systems that are capable to transmit higher data rates over larger geographical areas are not fulfilling the QOS needs. WiMAX is a substitute to wired DSL technology for lastmile solutions for providing backhaul services, thus increasing the data rate for large area. IEEE standard 802.16e provides fixed, nomadic, and mobile wireless broadband connectivity without the need for direct lineof- sight with the base station. This makes user to get all new and emerging services

such as Video on Demand (VoD), Internet Protocol Television etc at the required place. This paper analyses the Bit Error Rate (BER) throughput performance of WiMAX for AWGN channel.

\section{WIMAX NETWORK ARCHITECTURE}

WiMAX architecture comprises of several components but the basic two components are BS and SS. Other components are MS, ASN, CSN and CSN-GW etc. The WiMAX Forum's Network Working Group has developed a network reference model according to the IEEE 802.16e-2005 air interface to make sure the objectives of WiMAX are achieved. To support fixed, nomadic and mobile WiMAX network, the network reference model can be logically divided into three parts.

\subsection{Base Station (BS)}

The BS provides connection between operator networks and wireless subscriber devices. To enable wireless communications consists of antennas, transceivers, and other electromagnetic waventransmitting equipments.

\subsection{Subscriber Station (SS)}

Also called Mobile Station (MS). The SS is the user that needs to use services while in motion at vehicular speed. These SS are battery operated compared to the fixed station. Generally mobiles and laptops are used as SS.

\subsection{Access Service Network (ASN)}

It is owned by NAP, formed with one or several base stations and ASN gateways (ASN-GW) which creates radio access network. It provides all the access services with full mobility and efficient scalability. Its ASN-GW controls the access in the network and coordinates between data and networking elements. ASN-GW performs traffic management function within the ASN. 


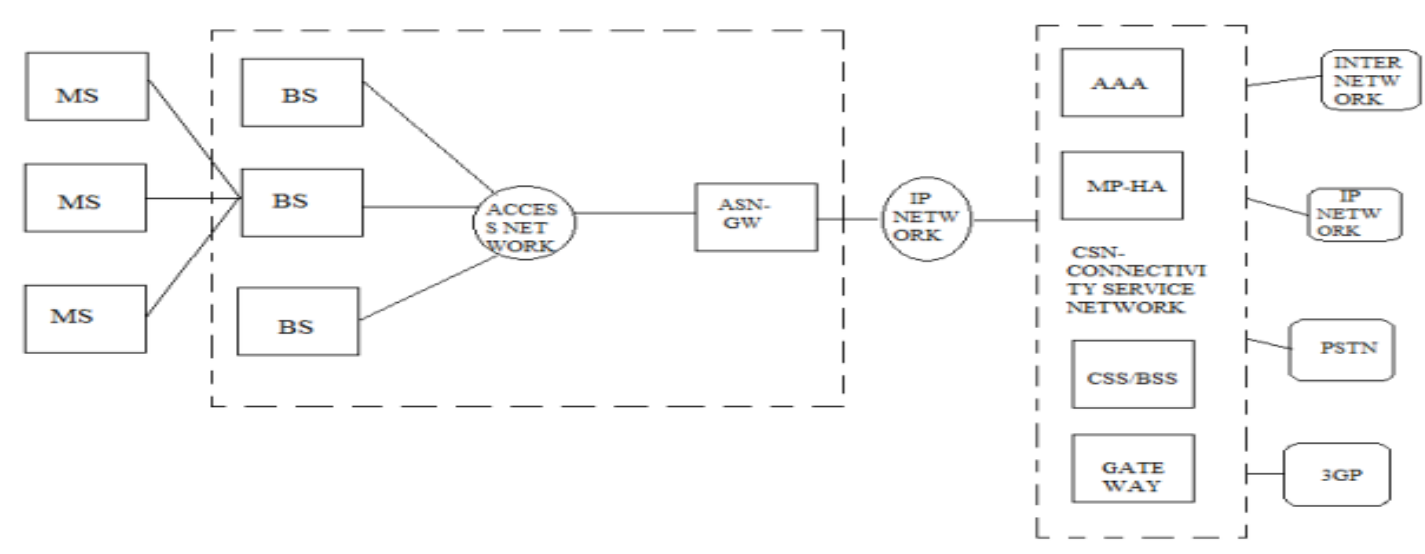

Fig: Wimax network Architecture

\subsection{Connectivity Service Network (CSN)}

Provides IP connectivity to the Internet or other public or corporate networks. It also applies per user policy management, address management, location management between ASN, ensures QoS, roaming and security.

\section{WIMAX COMPRISES OF TWO MAIN PARTS}

3.1.WiMAX base station

3.2. WiMAX receiver

\subsection{WiMAX base station}

It is often called WiMAX tower or booster. The base station broadcasts radio frequencies to the receiver end.

- Responsible for: Providing air interface to the MS and it performs in MAC and PHY.

- Additional functions: Frequency reuse, handoff, tunnel establishment, QoS \& classification of traffic etc.

- Management: Session management, bandwidth management for uplink and downlink and multicast group management etc.

- Practical Face: Tower in outdoor environment and electronic equipment in indoor environment.

\subsection{WiMAX receiver:}

WiMAX receiver receives the radio frequency from the WiMAX base station and makes sure the connectivity of WiMAX network is in range.

- Responsible for: Providing connectivity between subscriber equipment (such as mobile phone or laptop) and a WiMAX base station

- Additional function: Packet priority, network interoperability and QoS .

- Connection: Backhaul, high speed microwave link which is also referred to a connection between core network and WiMAX system.

- Provides User: VoIP, multimedia and Internet access and many mobile applications

- Practical face: Customer Premises Equipment (CPE) for indoor and outdoor purposes.

\section{WIMAX SIMULATION MODEL}

The WiMAX simulator presented in this paper had been implemented in MATLAB. The functional stages had been mainly design by using SIMULINK in MATLAB R2011a version. 


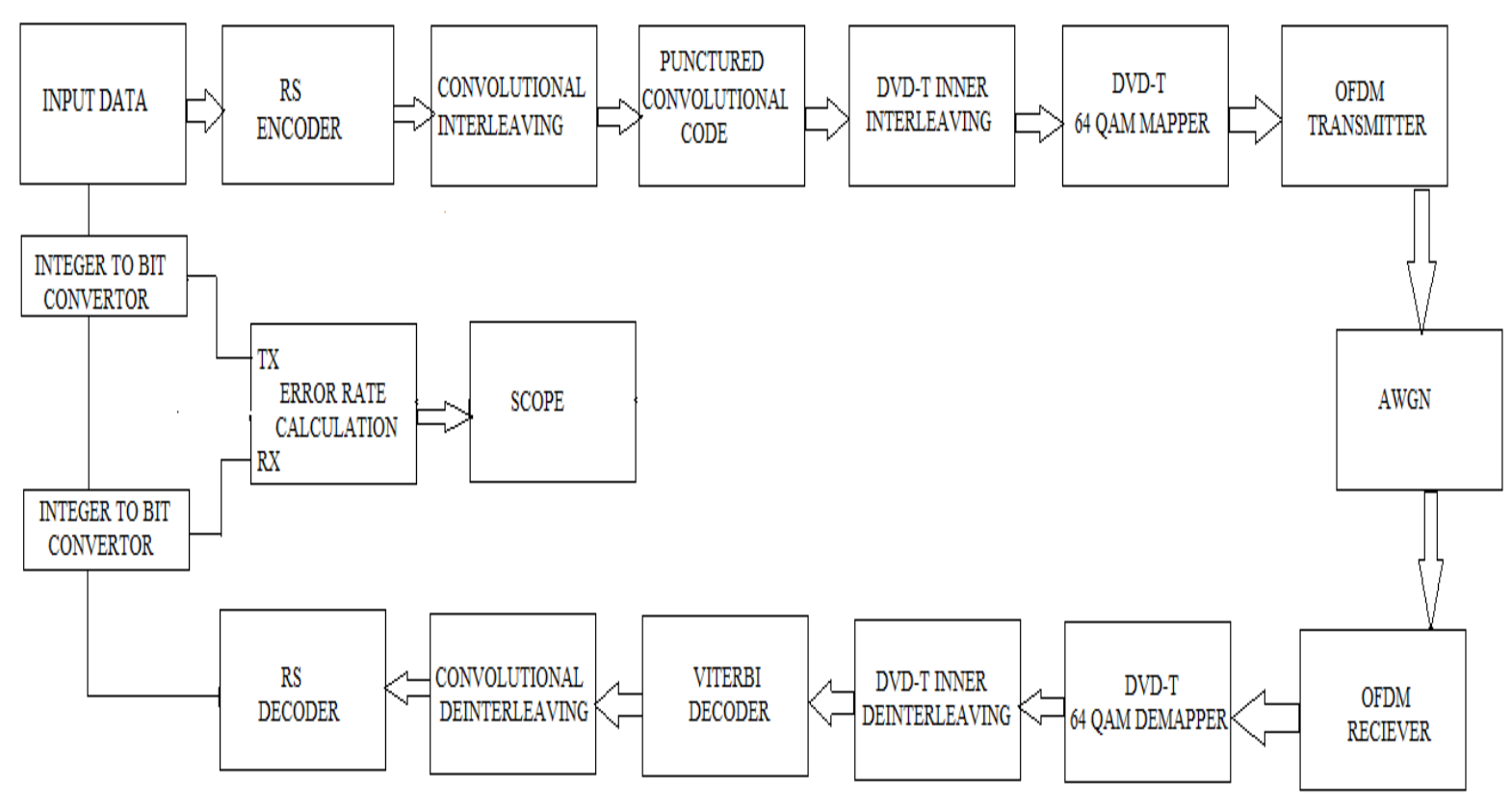

Fig: WIMAX SIMULATION MODEL

The DVB-T (Digital Video Broadcasting - Terrestrial) for the terrestrial transmission to fixed, portable and mobile receivers. The DVB-T Standard specifies the framing structure, channel coding and modulation for digital terrestrial broadcasting. The following processes are applied to the data stream :

- $\quad$ outer coding (i.e. Reed-Solomon code);

- $\quad$ outer interleaving (i.e. convolutional interleaving);

- inner coding (i.e. punctured convolutional code);

- $\quad$ inner interleaving (i.e. mapping and modulation);

- Orthogonal Frequency Division Multiplexing (OFDM) transmission.

\subsection{Outer Coding}

The Reed-Solomon encoding is mainly used to recover the main signal if it is distorted. The properties of Reed-Solomon codes make them suitable to applications, where errors occur in bursts. A Reed-Solomon code is specified as RS (n, k, t) with l-bit symbols. This means that the encoder takes $\mathrm{k}$ data symbols of 1 bits each and adds $2 t$ parity symbols to construct an $\mathrm{n}$-symbol codeword. Reed-Solomon code is RS $(204,188, t=8)$ encode.

\subsection{Outer Interleaving}

Convolutional byte-wise interleaving with depth $I=12$ is applied to the error protected 204 bytes packets. The interleaver is composed of $I=12$ branches, cyclically connected to the input byte stream by the input switch. Each branch $j$ shall be a First-In, First-Out (FIFO) shift register, with depth $j * M$ cells where $M=$ $17=N / I, N=204$.

\subsection{Inner Coding:}

Puncturing process is mainly used to convert a long bit stream data into short bit stream. It compress the long bit data. If we pass any balloon through a narrow pipe Puncturing is the process of systematically deleting bits from the output stream of a low-rate encoder in order to reduce the amount of data to be transmitted, thus forming a high-rate code. The bits are deleted according to a perforation matrix, where a "zero" means a discarded bit. There are different types of puncturing process like as $1 / 2$ rate, $2 / 3$ rate, $3 / 4$ rate, $5 / 6$ rate. In this model we have used puncture process $2 / 3$.

\subsection{Inner Interleaving}

Inner interleaving block consists of bit-wise interleaving followed by symbol interleaving. Both the bitwise interleaving and the symbol interleaving processes are block-based. Bit-wise interleaving input is demultiplexed (mapped to output modulation symbols) into $v$ sub streams, where $v=2$ for QPSK, $v=4$ for 16QAM, and $v=6$ for 64-QAM. Each sub-stream is then interleaved in the interleaver with own interleaving sequence - permutation function. The bit interleaving block size is 126 bits and is the same for each interleaver. 
Symbol interleaving is performed at bit-wise interleaved substreams. The purpose of the symbol interleaver is to map $v$ bit words onto the 1512 (in $2 \mathrm{~K}$ mode) or 6048 (in $8 \mathrm{~K}$ mode) active carriers per OFDM symbol.

\subsection{Ofdm}

Now we have to convert our data into OFDM symbol. For this reason we have to take the data as row format then add pilot signal and training signal with our data then use a vertical concatenation to make an OFDM symbol consists of data, pilot signal and training sequence and guard band. We have use complex function because of after modulation our data transform into complex form. The main purpose of training to generate guard band we have create a complex null vector. The main purpose to use guard band to prevent inter symbol interference (ISI). Then we have used a Matrix concatenation as an assembler. It is used to create an OFDM symbol and put the Sub carrier into that sequentially. Here we have set the matrix concatenation into vertical catenation format as our data in OFDM symbol is in vertical form.

\section{EXPERIMENTAL RESULTS}

The test was carried out on the WiMAX Simulation model.The performance is displayed in the in terms of the BER versus transmitted signal. We get the value of BER is .4991.

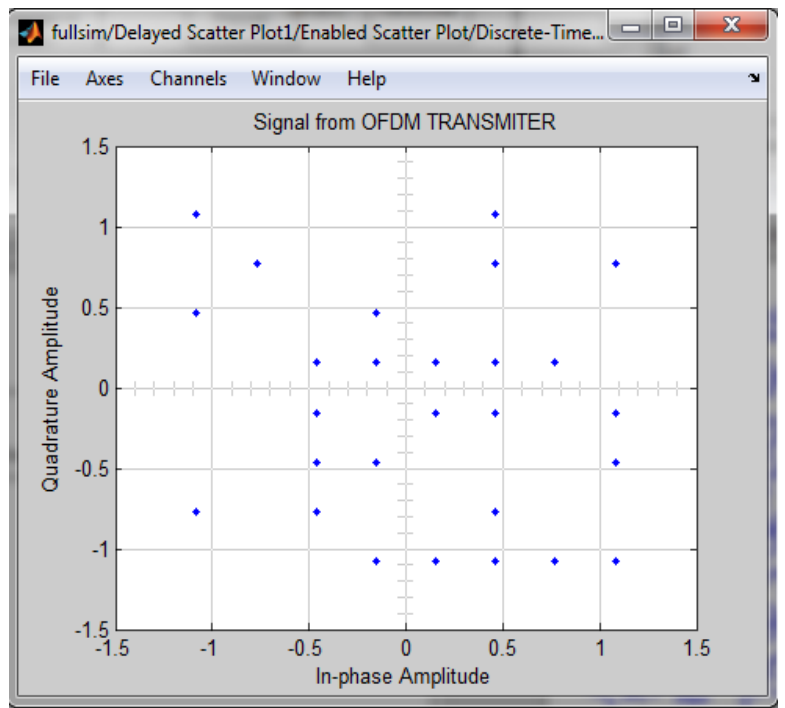

Transmitted Signal

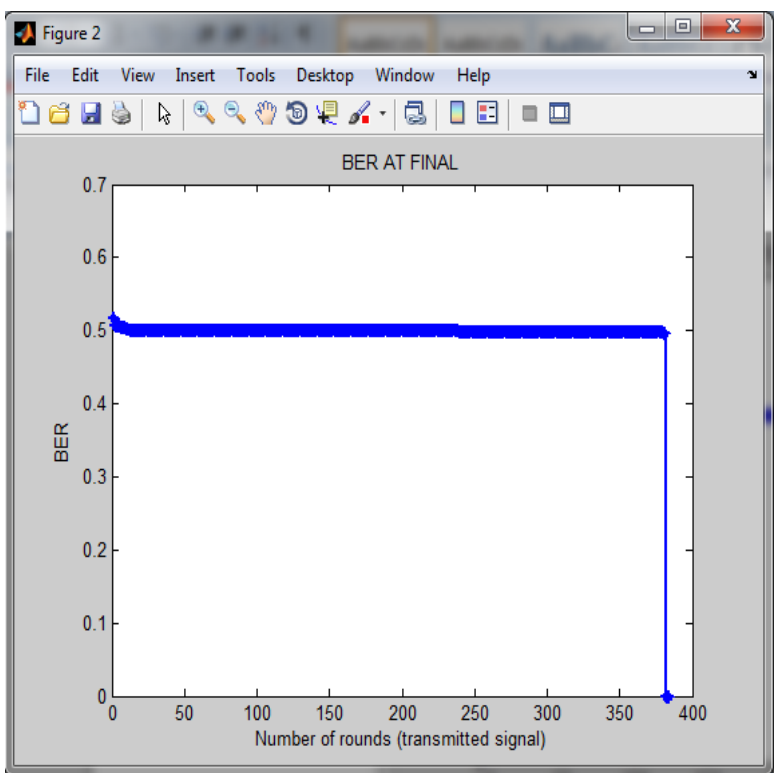

BER at final

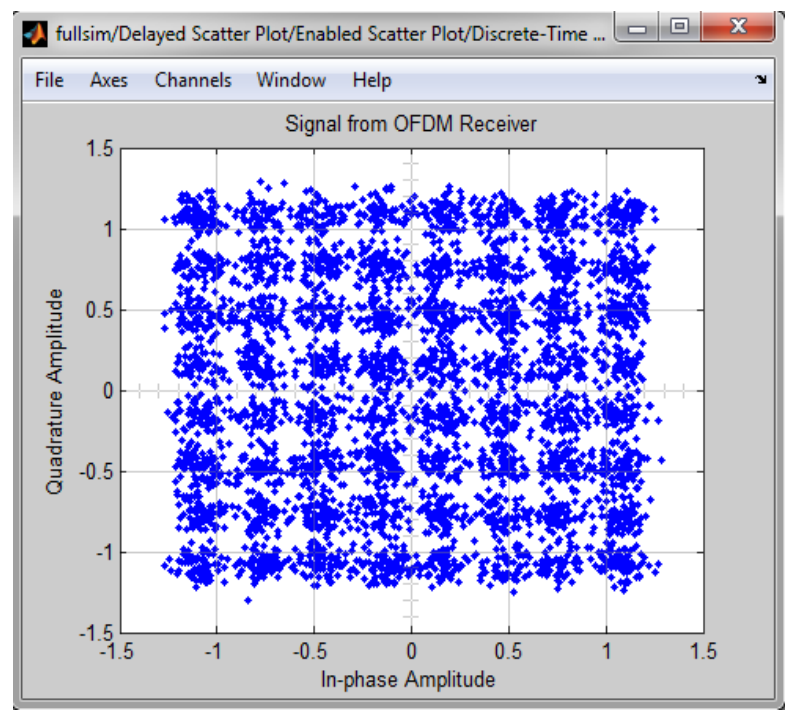

Received Signal

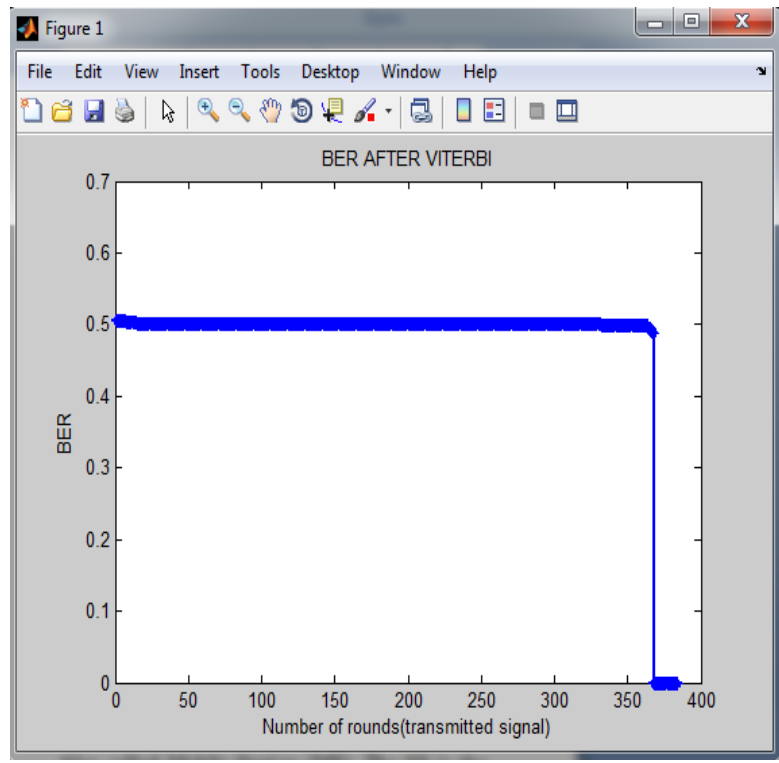

BER at Viterbi 


\section{CONCLUSION}

WiMAX technology brought revolution in both fixed and mobile wireless communication. In present communication world, wireless communication does not mean only data and voice transmission. It also supports high data rate transmission which supports various types of service like voice, data, multimedia. Presented implementation of DVB-T channel coder and decoder and results of its simulation in Matlab and error rates after corresponding error correction. However, simulated error rates are influenced by finite data length in opposite to continuous data stream in real digital television broadcast. So it can fulfill the demand of the present end users. Wi-Fi system is widely being used in the first world countries. WiMAX embedded devices support the Wi-Fi standards. So the people who are using Wi-Fi can easily switch to WiMAX technology. Moreover in the developing countries where high data rate wireless communication infra structure is not strong enough. WiMAX can be a good solution for these countries which is more secured, reliable and cheap. For these reasons the user of this technology is increasing day by day. As WiMAX is the latest technology and better solution in the wireless communication world, we have chosen this technology for our thesis.

\section{REFERENCES}

[1] J. G. Andrews, A. Ghosh, R. Muhamed,"Introduction to Broadband Wireless, in Fundamentals of WiMAX: understanding broadband wireless networking”, Prentice Hall, 2007.

[2] A. Roca, "Implementation of WiMAX simulator in Simulink", Engineering Institute-Vienna, February 2007

[3] IEEE Standard for Local and Metropolitan Area Networks Part 16,"Air Interface for Fixed broadband Wireless Access Systems", IEEE Computer Society.

[4] Hassan Yaghoobi, “Scalable OFDMA Physical Layer in IEEE 802.16 WirelessMAN”, Intel Communications Group, Intel Corporation. 2004.

[5] M.A. Hasan,"Performance Evaluation of WiMAX/IEEE 802.16 OFDM Physical Layer", Master of Science in Technology, Espoo, June 2007.

[6] Loutfi Nuaymi,“WiMAX Technology For Broadband Wireless Access”, Jhon willey publication, 2007.

[7] Eduardo Flores Flores, Raul Aquino Santos, Victor Rangel Licea, Miguel A. Garcia-Ruiz,"MAC layer Mechanism for Wireless WiMAX Networks with Mesh Topology", Electronics, Robotics and Automotive Mechanics Conference 2008.

[8] Fan Wang, Amitava Ghosh, Chandy Sankaran, Philip J. Fleming, Frank Hsieh, Stanley J. Benes, "Mobile WiMAX Systems: Performance and Evolution", IEEE Communications Magazine, October 2008. 